\title{
Influence of internal imperfections on the fatigue resistance of cast steel - testing methodology
}

\author{
Sven Nagel ${ }^{1,1}$, Christof Rauber $^{2}$, Ines Veile ${ }^{2}$, Peter Knödel ${ }^{1}$, Thomas Ummenhofer ${ }^{1}$, \\ ${ }^{1}$ KIT Steel \& Lightweight Structures, Karlsruhe, Germany \\ ${ }^{2}$ Fraunhofer Institute for Nondestructive Testing IZFP, Saarbrücken, Germany
}

\begin{abstract}
Tensile fatigue specimen of G20Mn5 and G22NiMoCr5-6 were tested to quantify the influence of internal defects on the fatigue resistance of cast steel components. Defects with varying sizes, geometric shapes and distribution were enforced by influencing the solidification and recorded by computer tomography (CT). Besides the characteristics of the detected cavities, the surrounding fungoid microstructure is classified and evaluated. Later the specimens were tested under cyclic tension and $\mathrm{S} / \mathrm{N}$-curves are derived. These data form the basis for extensive numerical simulations of the damage process and the crack growth of every individual specimen. Both processes are affected by the local multiaxial stress states and have their origin in the inside of the specimen. For validation, knowledge of the crack initiation time and propagation properties are essential. Therefore, all specimens respectively the properties of the internal defects are monitored during testing with three different state-of-the-art non-destructive testing (NDT) methods. Background and application of these NDT techniques are described within this paper. Finally, fracture surface analyses show different failure modes and provide further information for model validation.
\end{abstract}

\section{Introduction}

Cast steel components are used in several industrial sectors such as construction industry, transportation or mechanical engineering. In theory, they are well suitable for cyclic loading due to their smooth contours. Nearly any geometry can be adapted to the individual stress distribution to reduce high stress concentrations as they appear in welds or at sharp notches. Along with the casting process, especially during solidification of the steel, different casting defects occur. Former research [1-3] has shown a reduction in lifetime as these defects cause multiaxial stress fields and act as notches which could have the shape of sharp cracks. To which extend the positive or negative effects dominate the overall behavior is not quantified yet. Reason to hope are millions of cast components, which behave well in service. During the last decades foundry research mostly focused on the prediction and avoidance of casting defects. In general, this results in higher expenses in the casting process causing uneconomic and oversized components. Independent of the industrial sector these effects are not considered in the design standards (offshore industries [4], mechanical engineering [5] and

\footnotetext{
Corresponding author: sven.nagel@kit.edu
} 
building structures [6]). In most cases, highest quality classes are requested by default, independent of being feasible or not are requested by default and $100 \%$ NDT is required. A quantified and targeted consideration of the defects' influence on the component's fatigue design resistance is yet missing for general applications.

To fill this gap in knowledge and design guides the research project "ErStaGu" [7] has been set in place. The scope of the present article is the description of fatigue tests on tensile specimen with internal defects. These tests form the basis for numerical simulations and the improvement of design guidelines. Special evaluation techniques such as state-of-the-art NDT methods are required due to the variety and location of the casting imperfections. After introducing the specimens' design, first results of the reconstructed defects by CT are presented. Later the NDT methods are introduced in detail and their combination is discussed. Finally, the test results are evaluated considering fracture surface analysis. Besides the investigations presented within this paper, material characterization is done based on monotonous tensile tests, low-cycle fatigue tests, incremental step tests, crack growth tests and high-cycle fatigue tests. For these investigations, blocks of material without imperfections have been casted. These tests form the basis of advanced material and damage models used in numerical simulations with different abstraction levels [8]. Starting with the consideration of both, crack initiation and crack growth process, at the precise geometry of each defect, the decisive phenomenon are going to be described and understood. On the lowest modeling level the defects are reduced to simple geometries and only certain phenomena are considered. The third type of tests are full-scale component tests to investigate the interaction of geometric notches and casting defects as well as the influence of the wall thickness. After depicting the relevant influences numerically, parameter studies and worst-case scenarios are investigated to deduce design procedures.

\section{Testing}

\subsection{Specimen}

The aim was to generate tensile specimen with reproducible internal defects. Following the same goal, [9] used round cast steel bars with thickened areas to enforce shrinkage porosity of different size and shape for monotonous tensile tests. Reevaluating these specimens, two geometries were selected and redesigned by casting process simulations. The resulting cast geometry is given in Fig. 1 a). The first type is without any thickened parts of the specimens' center causing pores along the centerline. In further explanations these specimens are referred to as $(\mathrm{N}=$ no increase). The second type had an expansion that induced compacted shaped pores with a reduction of the net cross section between $10 \%$ to $20 \%$. (I) is used for further reference. To cover a broad range of materials used in structural components a widely used medium strength G20Mn5+QT [10] and a high strength G22NiMoCr5-6 [11] were investigated. Specimen of both materials were casted by two foundries using different molding techniques. The mechanical properties according to their inspection certificates are given in Table 1. In a second step, the actual test geometry was machined from these cast bars. To neglect effects of the specimen geometry, a spiral shape as shown in Fig. 1 b) was selected. 


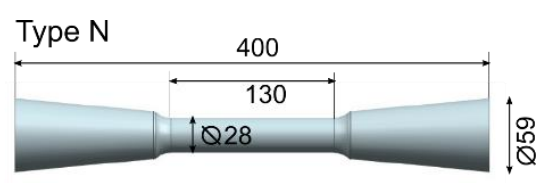

Type I

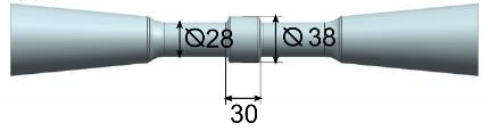

a)
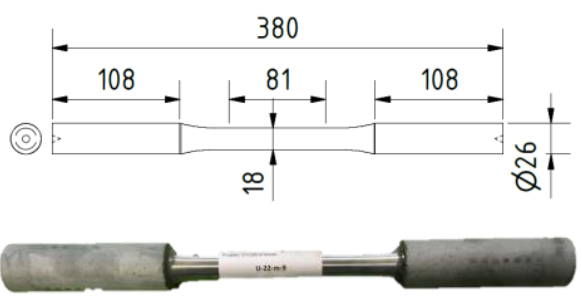

b)

Fig. 1. a) Geometry of cast bars b) cast process simulation to determine the thickening and resulting defect sizes b) final geometry

Table 1. Mechanical properties according to inspection certificates

\begin{tabular}{|l|l|l|l|l|}
\hline Material & $R_{\text {eh }}[\mathrm{MPa}]$ & $\mathrm{R}_{\mathrm{m}}[\mathrm{MPa}]$ & $\mathrm{A}[\%]$ & $\mathrm{KV}[\mathrm{J}]$ \\
\hline G20Mn5 & 360 & $500-650$ & 22 & $27 \mathrm{~J}$ at $-20^{\circ} \mathrm{C}$ \\
\hline Foundry 1 & 401 & 583 & 24,4 & $71 / 68 / 64$ \\
\hline Foundry 2 & 449 & 617 & 22,3 & $53 / 61 / 59$ \\
\hline G22NiMoCr5-6 & 825 & $930-1080$ & 10 & $27 \mathrm{~J}$ at $-40^{\circ} \mathrm{C}$ \\
\hline Foundry 1 & 849 & 979 & 15,1 & $27 / 28 / 28$ \\
\hline Foundry 2 & 963 & 1065 & 14,1 & $43 / 35 / 34$ \\
\hline
\end{tabular}

\subsection{Computer tomography (CT)}

The internal defects were recorded by 3D CT scans. The specimen were radiated by a 225 $\mathrm{keV} \mathrm{x}$-ray source and the local absorption is used to form a projection of varying material density. To reconstruct a full 3D geometry projections from all sides are necessary. Further information concerning this technique is given in [12]. In this case, a resolution of 88 $\mu \mathrm{m} /$ voxel was considered to be sufficient. The output of these scans is the $3 \mathrm{D}$ reconstruction file, which is used for pore analysis and is transformed into slices to build up a finite element model [13] of high resolution as well as smoothed stl-file based surface models. Within the pore analysis the number, size, projection surface and further characteristic values describing the geometric properties of the pores were evaluated. In addition, the plane of maximum cross-sectional reduction was determined. Fig. 2 shows typical pore representations. $\mathrm{N}$ type specimen contained a lot more but small pores, whereas the internal structure of the I type specimen were mostly dominated by a single larger pore. This statement could be quantified as follows: I type contained 20 pores on average, in which the volumetrically largest pore makes up $95 \%$ to $99 \%$ of the total pore volume. The amount of pores in $\mathrm{N}$ type specimen is 50 in average and can go up to 200 pores in individual cases. The dominance of one pore gets lost in many cases and different defects of the similar size exist. The largest pore is $10 \%$ to $90 \%$ of the total pore volume. Comparing maximum material reductions in planes perpendicular to the load direction $\mathrm{N}$ type specimen contained defects in the size of $0.5 \%$ to $9.5 \%$ of the bars cross-section. This value was between $5 \%$ and $15 \%$ for I type specimen. In these evaluations no substantial influence of the material or molding technique concerning the discussed properties were detected. 


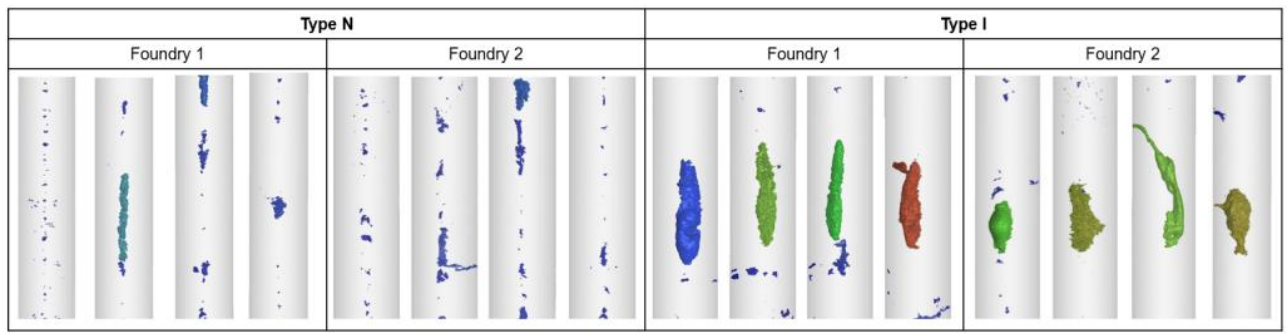

Fig. 2. CT-Scans of $\mathrm{N}$ and I specimen show different pore shapes and sizes

Former research $[9,14]$ has identified a fungoid microstructure surrounding some of the cavities. Fig. 3 shows a pore including the described microstructure on the crack surface of a (I) specimen recorded by a scanning electron microscope (SEM). The influence of these areas on the crack initiation is not yet understood. Either parts of these areas act as sharp cracks so, that no classical damage process takes place, or the surrounding pliant zone smooths the stress concentrations along the pores edges and enlarges the fatigue resistance. Besides, it is not yet clarified, whether these areas participate in the load transfer. To get a better understanding of the mechanisms taking place in these areas the pore analysis was done with two different settings. The first captured the pure cavity; the second included the fungoid microstructure. The following evaluations focus on the I type specimen, since it was not possible to distinguish between a cavity and the fungoid microstructure in the CT data of the small pores of the $\mathrm{N}$ type specimen. In these cases, an analysis of the fracture surface was more purposeful. In Fig. 3 c) both evaluations - only the cavity and the complete defect region including the fungoid microstructure - are sown graphically.

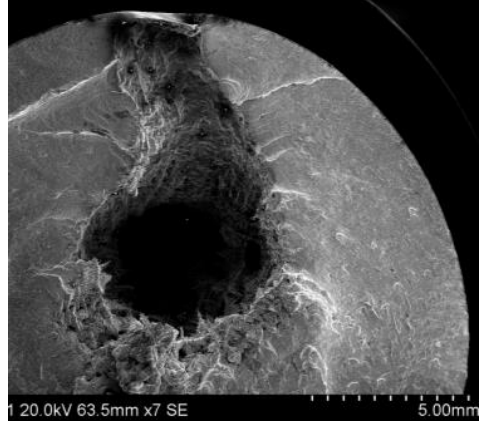

a)

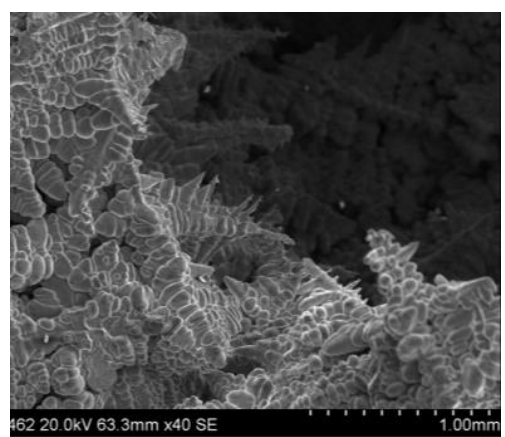

b)

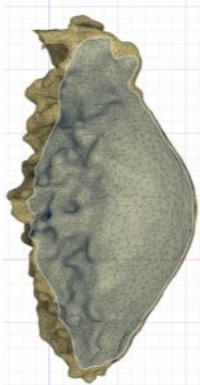

c)

Fig. 3 a) overview and b) detailed STM record of a casting defect and the surrounding fungoid microstructure [Recorded at Fraunhofer IWM Freiburg]. c) Reconstruction of the pore (light color) and the complete volume including the fungoid microstructure (darker color)

Evaluating the pore analysis of the CT-Scans, the cavity volume only makes up between $55 \%$ and $75 \%$ of the complete volume. Considering the defects surface an increase of $40 \%$ to $80 \%$ was determined while, the shape becomes less spherical and less compact.

\subsection{Fatigue Testing}

The testing of all specimen was done on a $400 \mathrm{kN}$ high-frequency pulsator. All tests were performed under cyclic tension with a stress ratio $\mathrm{R}=0.1$. The applied loads where chosen to cover nominal stress amplitudes between 150 and $570 \mathrm{MPa}$ resulting in 1E4 to 1E7 cycles 
to failure. Prior to testing the clamping area of all specimen were embedded in a rasin to ensure an electric and tonal isolation from the test setup. During testing, the NDT methods described in section 3.1 were used to determine the origin and time of crack initiation, possible competition of multiple cracks and their respective growth rate.

\section{$3 \quad$ Non-destructive testing}

\subsection{Applied NDT methods}

Electromagnetic ultrasound (EMAT):

Electromagnetically excited ultrasound presents an advantageous alternative to conventional ultrasound testing, especially in rough environments or when special wave modes are to be excited. In principle, an electromagnetic acoustic transducer (EMAT) is consisting of a magnet and a radio frequency coil. The ultrasonic sources are induced in the material surface by alternating electromagnetic fields. From these sources, an ultrasonic wave is generated in the specimen. The attenuation and the time of flight of the ultrasonic wave are typical parameters to be evaluated. General information concerning this technique is given in [15, $16]$.

\section{Acoustic emission (AE):}

Elastic waves emitted by the formation of microscopic cracks or structural changes in solid state bodies are referred to as acoustic emission (AE). AE is used as a method for the detection and the valuation of crack growth arising and structural changes. Crack growth and structural changes cause elastic waves. Sensors can measure these elastic waves at the surface of the solid state bodies [17].

Magnetic flux leakage (MFL):

MFL inspection using high sensitivity magnetic sensors present an important alternative to conventional magnetic particle testing. Test specimens can be magnetized by a permanent magnet or an electromagnet before the test as well as throughout the test. Flux leakage occurs at the surface of the specimen in places with cracks perpendicular to the magnetization direction and this leakage can be measured [18].

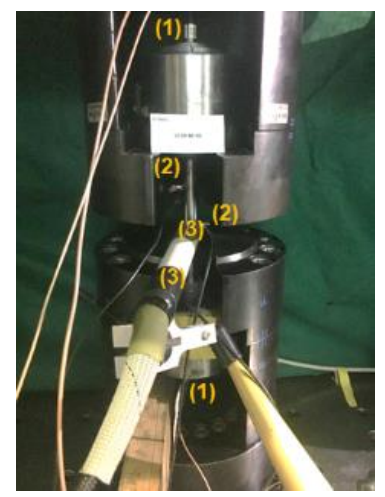

a)

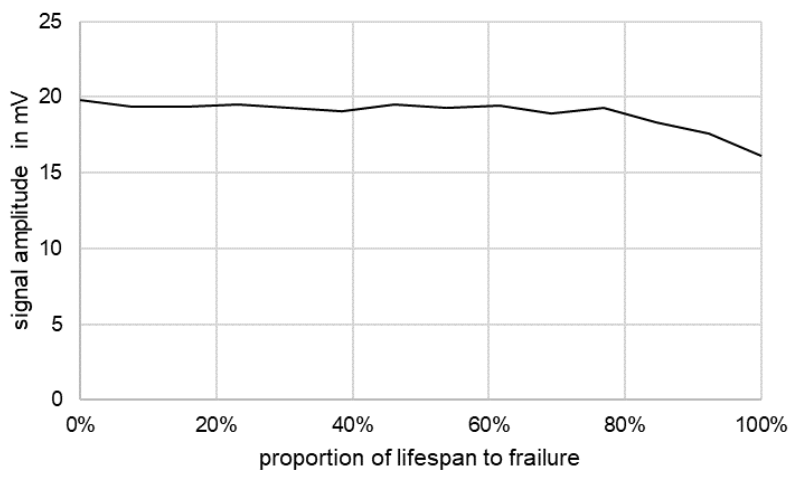

b)

Fig. 4. a) Specimen under test conditions with EMAT (1), AE (2) and differential MFL (3) sensors b) decreasing amplitudes in an EMAT measurement 


\subsection{Non-destructive detection of the crack initiation time and the crack propagation}

State-of-the-art methods do not contain a robust solution for monitoring internal defects during fatigue tests. In order to detect the instant of crack initiation and to monitor the crack propagation, the three presented NDT methods were investigated and a combination of significant parameters extracted from the recorded signals resulting from all applied methods was evaluated. Regarding ultrasonic testing with EMATs, the time of flight and the amplitude of the ultrasonic wave were analyzed. Regarding MFL testing, the crack opening (which is related to the crack propagation) causes an increasing magnetic flux leakage that can be recorded at the specimen's surface. Thus, the change of the magnetic field measured at the specimen's surface over the testing time is analyzed. Using AE, acoustic events occurring in the specimen during testing are detected. In this context, the frequency and intensity of these events are investigated as they allow conclusions about crack initiation processes. In addition, the installation of three AE sensors with a specific spatial distribution generally enables the localization of one or multiple cracks. The applied methods complement each other as initial changes in the microstructure can be detected with EMAT. Resulting crack initiation could be detected with all these NDT methods and the crack opening was detected most clearly with MFL measurements. Through that, there is a high probability that all changes in the specimen can be monitored by simultaneous use of these three methods and a subsequent combination of the individual results. Figure 4 a) shows one of the specimens under test conditions with the mentioned sensors. First evaluations of the recorded data show the expected effects. As an example, the in-situ recorded amplitude of an EMAT measurement is shown in Figure 4 b). The amplitude decreased while significant changes occurred in the specimen structure during the last third of the test. At this point further measurements were executed and the recorded data was analyzed in detail. It is subject of ongoing research, if a combination of these in-situ applied methods allows for the detection of the crack initiation and the monitoring of the crack propagation.

\section{$4 \mathrm{~S} / \mathrm{N}$ curves and test results}

\subsection{Raw Data}

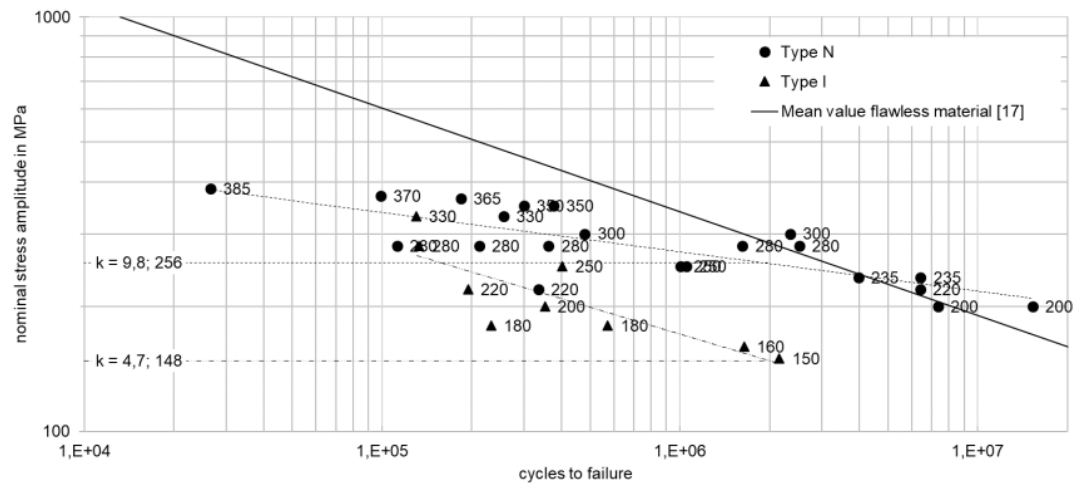

Fig. 5. Nominal S/N-curves (G20Mn5) without considering the internal defects quantitatively. FAT class for mean value and slope. The tested mean values of flawless material according to [19] are given as reference. 
Fig. 5 presents the raw test results for $\mathrm{N}$ and I type specimen of G20Mn5. The stress amplitudes are related to the nominal cross-section without considering the defects. In general, a lower fatigue resistance is determined for the I type specimen. In all cases a large variance and scatter is evident. A preliminary fatigue class and slope of the mean value $\mathrm{S} / \mathrm{N}$ curve is given in Fig. 5.

\subsection{Fracture surface analysis}

After testing, each fracture surface was analyzed on different scales. With visual examinations charactering (Fig. 6) the topology of one or several crack surfaces. Records taken with a microscope were used to measure sizes of cavities, fungoid microstructure and the area and shape of the crack growth as well as the residual fracture face (Fig. 7). SEM scans did provide information of high resolution (Fig. 3) for selected specimen. Comparing the specimen in Fig. 6 a) characteristic for low stress amplitudes and high cycles to failure and b) characteristic for high stresses and less cycles, the area of the residual fracture face differ significantly as expected. In both cases these areas are dominated by shear lips and the expected constriction for the higher loads. But also, the topology is different in the area of the crack surface. In the cases of lower stress amplitudes, flat surfaces occur whereas under higher loads there are more irregularities. Fig. 6 c) represents a specimen in which cracks started and grew in different planes. When reaching a critical length, the specimen failed by concrescence of these cracks. This phenomenon was detected independent of load levels and need to be investigated in more detail by evaluating the NDT results as well as by simulations.

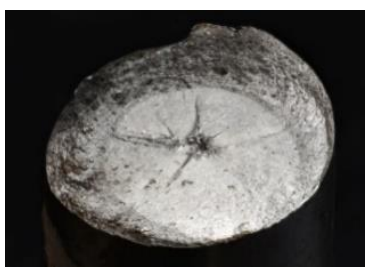

a)

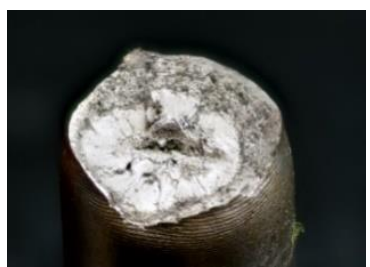

b)

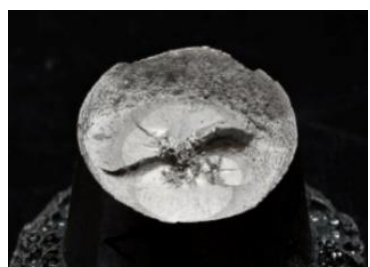

c)

Fig. 6. Visual examination of the fracture surface for a) $\Delta \sigma_{\text {nominal }}=220 \mathrm{MPa}-6.400 .000$ cycles b) $370 \mathrm{MPa}-100.000$ cycles c) $235 \mathrm{MPa}-4.000 .000$ cycles

Apart from the differences resulting from the load level and cavity size, the fracture surface differs significantly for defects reaching the surface. Fig. 7 b) (case 1 for further references) and those completely remain in the inside of the specimen Fig. 7 a) (case 2). The majority of the samples are case 2 . In case 2 the crack growth is clearly visible and of an elliptical shape. In case 1 on the other hand, this region passes on to the residual fracture face and does not show a clear geometric shape. Evaluating the visible changes of microstructure around the cavities, a load-carrying cross-section and resulting net stress amplitudes are determined. 


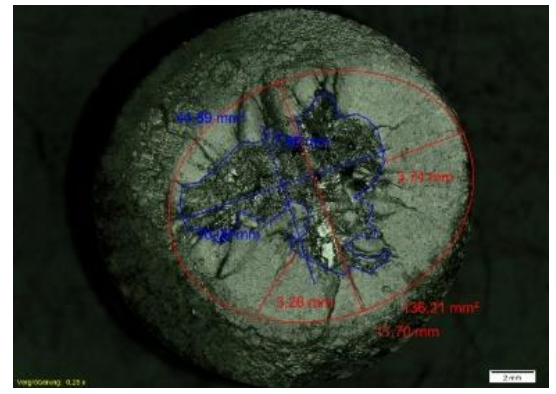

a)

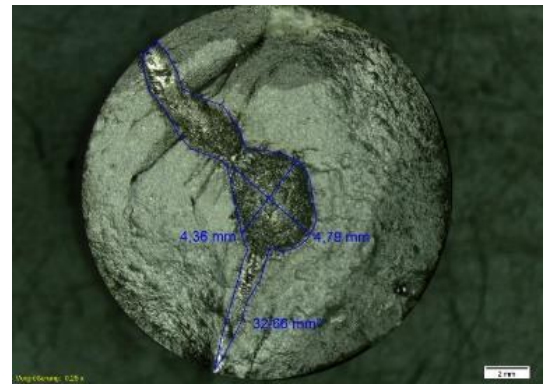

b)

Fig. 7. Microscopic evaluation of the fracture surface a) pure internal defect $b$ ) internal defect reaching the surface

\subsection{Re-evaluation of the test results}

Considering the actual net section of each individual specimen by adding the knowledge of chapter 4.2, the wide scatter and irregularity of the raw data presented in Fig. 5 becomes clearly defined as shown in Fig. 8. a) independent of the type of specimen (I or N) two separated populations could be identified and assigned to the cases 1 and 2 of section 4.2 for the G20Mn5. All specimen of the lower group belong to case 1, the upper to case 2. Both evaluations show much smaller scatter. The associated slope and FAT-class $(\Delta \sigma$ for $N=2 \mathrm{E} 6$ cycles) are given within the diagrams. This separation of internal and external defects is not pronounced as clearly for the G22NiMoCr5-6.
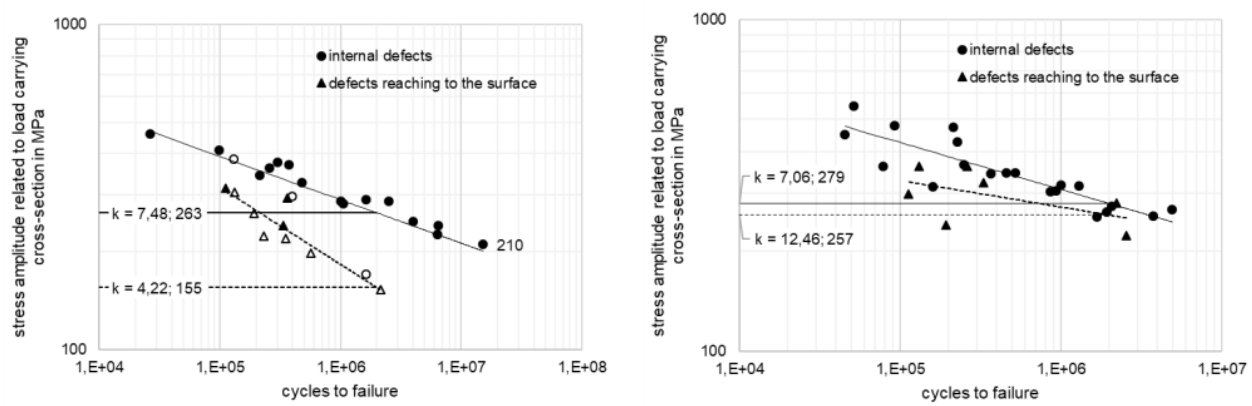

Fig. 8. S/N-curves for the actual net section a) G20Mn5 b) G22NiMoCr5-6

\section{References}

1. R.A. Hardin, C. Beckermann. Prediction of the Fatigue Life of Cast Steel Containing Shrinkage Porosity. Metallurgical and Materials Transactions, 40, 581-597 (2009).

2. E.S. Breznak, C. Vishnevsky, J.F. Wallace. The effect of internal shrinkage discontinuities on the fatigue and impact properties of cast steel sections, Steel foundry research foundation Rocky River Ohio, Rocky River, Ohio (1969).

3. A. Ma, J.V. Sharp, Fatigue design of cast steel nodes in offshore structures based on research data. Proceedings of the Institution of Civil Engineers - Water and Maritime Engineering. 112-126 (1997).

4. DNV-RP-C203, Fatigue Design of Offshore Steel Structures (2012-10). 
5. R. Rennert. FKM Richtlinie: Rechnerischer Festigkeitsnachweis für Maschinenbauteile aus Stahl, Eisenguss- und Aluminiumwerkstoffen (engl. Calculated proof of strength for machine components). VDMA-Verl. Frankfurt (2012).

6. DIN EN 1993-1-8/NA. Design of steel structures - Part 1-8: Design of joints - German National Annex (2010-12).

7. S. Nagel, I. Veile, M. Farajian, T. Ummenhofer. ErStaGu Design of fatigue-stressed cast steel components under consideration of production-related discontinuities, IGF No.: $19691 \mathrm{~N}$ research project funded by the German Federal Ministry of Economics and Energy on the basis of a resolution of the German Bundestag (2017-2020).

8. S. Nagel, P. Knödel, T. Ummenhofer. Ultrakurzzeitfestigkeit von geschweißten Verbindungen unter mehrachsigen Beanspruchungszuständen (engl. Ultra-low cycle fatigue of welded joints under multi-axis stress states), 21. DAStForschungskolloquium, Kaiserslautern (2018).

9. M. Spannaus, Bemessung von Erzeugnissen aus Stahlguss unter vorwiegend ruhender Beanspruchung (engl. Design of cast steel under static load). KIT Scientific Publishing, Karlsruhe (2016).

10. EN 10293, Steel castings - Steel castings for general engineering uses, (2015-04).

11. SEW 520, High-strength cast steel with good weldability - Technical delivery conditions (2017-12).

12. T.M. Buzug, Computed Tomography From Photon Statistics to Modern Cone-Beam CT, Springer-Verlag, Heidelberg (2008).

13. P. Tempel, C. Eichheimer. Digitalisierung komplexer Volumendefektverteilungen am Beispiel von Stahlguss für die Festigkeitsbewertung unter quasi-statischer Zugbeanspruchung (engl. Digitization of complex volume defect distributions), Werkstoffwoche, Dresden (2017).

14. I. Veile, M. Farajian. IGF Report 469 ZN: Influence of production-related discontinuities on the material behavior of cast steel, Gießereitechnik e.V. (FVG), Düsseldorf (2017).

15. G. Hübschen, I. Altpeter, R. Tschuncky, H.-G. Herrmann (Eds.). Materials Characterization Using Nondestructive Evaluation (NDE) Methods. Woodhead Publishing, Elsevier, Amsterdam (2016).

16. I. Altpeter, R. Tschuncky, K. Hällen, G. Dobmann, C. Boller, A. Sorich, M. Smaga, D. Eifler. Early detection of damage in thermo-cyclically loaded austenitic materials, Studies in Applied Electromagnetics and Mechanics, 36, 130-139 (2012).

17. C.U. Große, Quantitative zerstörungsfreie Prüfung von Baustoffen mittels Schallemissionsanalyse und Ultraschall (engl. Quantitative non-destructive testing of building materials using acoustic emission analysis and ultrasound), Stuttgart University (1996).

18. K. Szielasko, A. Kloster, I. Altpeter, G. Dobmann, M. Kröning. Magnetische Streuflussprüfung mit GMR-Sensoren (engl. Magnetic leakage flux test with GMR sensors), DGZfP-Jahrestagung, Münster (2009).

19. Offshore Technology Report - OTH 92390 - Background to new fatigue guidance for steel joints and connections in offshore structures, Health and Safety Executive (1999). 